\title{
Unexpected worsening of progressive multifocal leucoencephalopathy following COVID-19 pneumonia
}

\author{
S. Borrelli ${ }^{1}$ (1) B. Dachy ${ }^{1} \cdot$ M-D. Gazagnes ${ }^{1} \cdot$ R. Du Pasquier ${ }^{2}$
}

Received: 20 January 2021 / Revised: 25 March 2021 / Accepted: 6 April 2021 / Published online: 19 April 2021

(c) Journal of NeuroVirology, Inc. 2021

\begin{abstract}
Progressive multifocal leucoencephalopathy is a serious side effect of natalizumab, a humanized monoclonal antibody approved for the treatment of multiple sclerosis. Here, we report a case of unexpected worsening of natalizumab-related progressive multifocal leucoencephalopathy following COVID-19. After natalizumab discontinuation, a slight neurological improvement was observed, but, two months later the patient was admitted to the hospital because of neurological deterioration and COVID-19 mild pneumonia. Except for SARS-CoV-2 infection, no other potential factors of neurological worsening were identified. Thus, we pose the hypothesis that SARS-CoV-2 was instrumental in the progressive multifocal leucoencephalopathy deterioration.
\end{abstract}

Keywords Progressive multifocal leucoencephalopathy $\cdot$ Natalizumab $\cdot$ COVID-19 $\cdot$ SARS-CoV-2 $\cdot$ Multiple sclerosis

Progressive multifocal leucoencephalopathy (PML) is a serious side effect of natalizumab (NTZ) (Bloomgren et al. 2012), a humanized monoclonal antibody against $\alpha 4$-integrin that is approved for the treatment of relapsing forms of multiple sclerosis (MS) (Polman et al. 2006; Rudick et al. 2006). PML is an opportunistic viral infection of the central nervous system (CNS) caused by reactivation and replication of the John Cunningham virus (JCV), which is characterized by a progressive multifocal disease of white matter (Bloomgren et al. 2012). Here, we report a case of unexpected worsening of NTZ-related PML after COVID-19.

A 50-year-old man with MS was treated with NTZ since 2009. Despite the JCV serology was highly positive (titer of 2.67) since 2017, the patient's personal choice was to remain on NTZ. In March 2020, dysarthria with the detection of new right frontal MRI lesion (Fig. 1a) and 61,500/ $\mathrm{ml} \mathrm{CSF} \mathrm{JCV} \mathrm{copies} \mathrm{led} \mathrm{to} \mathrm{the} \mathrm{diagnosis} \mathrm{of} \mathrm{PML.} \mathrm{CSF} \mathrm{pro-}$ tein and cell count were in normal ranges. Therefore, NTZ

\section{S. Borrelli}

serena.borrelli.sb@gmail.com;

serena.borrelli@chu-brugmann.be

1 Department of Neurology, Brugmann University Hospital and Free University of Brussels (ULB), Brussels, Belgium

2 Service of Neurology, Department of Clinical Neurosciences, Lausanne University Hospital (CHUV) and University of Lausanne, Lausanne, Switzerland was discontinued. A slight neurological improvement was observed, but, in April 2020, the patient was admitted to the hospital because of speech worsening, left hemiparesis, and hemianopsia. No fever or respiratory dysfunction was observed, but lung CT scan showed ground-glass opacities (Fig. 1b), and the nasopharyngeal SARS-CoV-2 antigen analysis was positive. Blood tests showed only slight inflammatory syndrome (C-reactive protein of $5.2 \mathrm{mg} / \mathrm{L}$ ), with mild neutrophilic leukocytosis (absolute leukocyte count of $11,940 / \mu \mathrm{L}, 57.7 \%$ neutrophil, and $28.1 \%$ lymphocyte) and increase in liver enzymes (ALT 50 U/L, AST 178 U/L). Thus, a diagnosis of mild COVID-19 pneumonia was made. No treatment was given. Brain MRI revealed PML lesion progression (Fig. 1c) with no gadolinium enhancement (Fig. 1d). A progressive neurological worsening was observed until May 2020, corresponding to an enlargement of the PML brain lesions (Fig. 2a) and the appearance of gadolinium enhancement (Fig. 2b). No corticosteroids were administered. Following the spontaneous healing of COVID19, a gradual clinical and radiological recovery (Fig. 2c, d) was observed starting June 2020. A second CSF analysis showed slight inflammatory signs (protein concentration of $0.49 \mathrm{~g} / \mathrm{L}$ and predominant lymphocyte cell count of 8.6/ $\mu \mathrm{L}$ ), a significant reduction in JCV replication (668 copies/ $\mathrm{ml}$ ) and a negative SARS-CoV-2 PCR. After 6 months in rehabilitation unit, the patient was discharged. The follow-up brain MRI performed in November 2020 showed one new 
Fig. 1 a Axial brain T2/Flairweighted MRI shows a right frontal lesion, mainly confined to the subcortical white matter, with relative sparing of the cortex, in March 2020. b Chest CT scan demonstrates basal pulmonary ground-glass opacities and consolidations, in April 2020. Axial brain T2/Flair-weighted MRI shows enlargement of the right frontal lesion (c) with no enhancement on brain postcontrast T1-weighted MRI (d), in April 2020
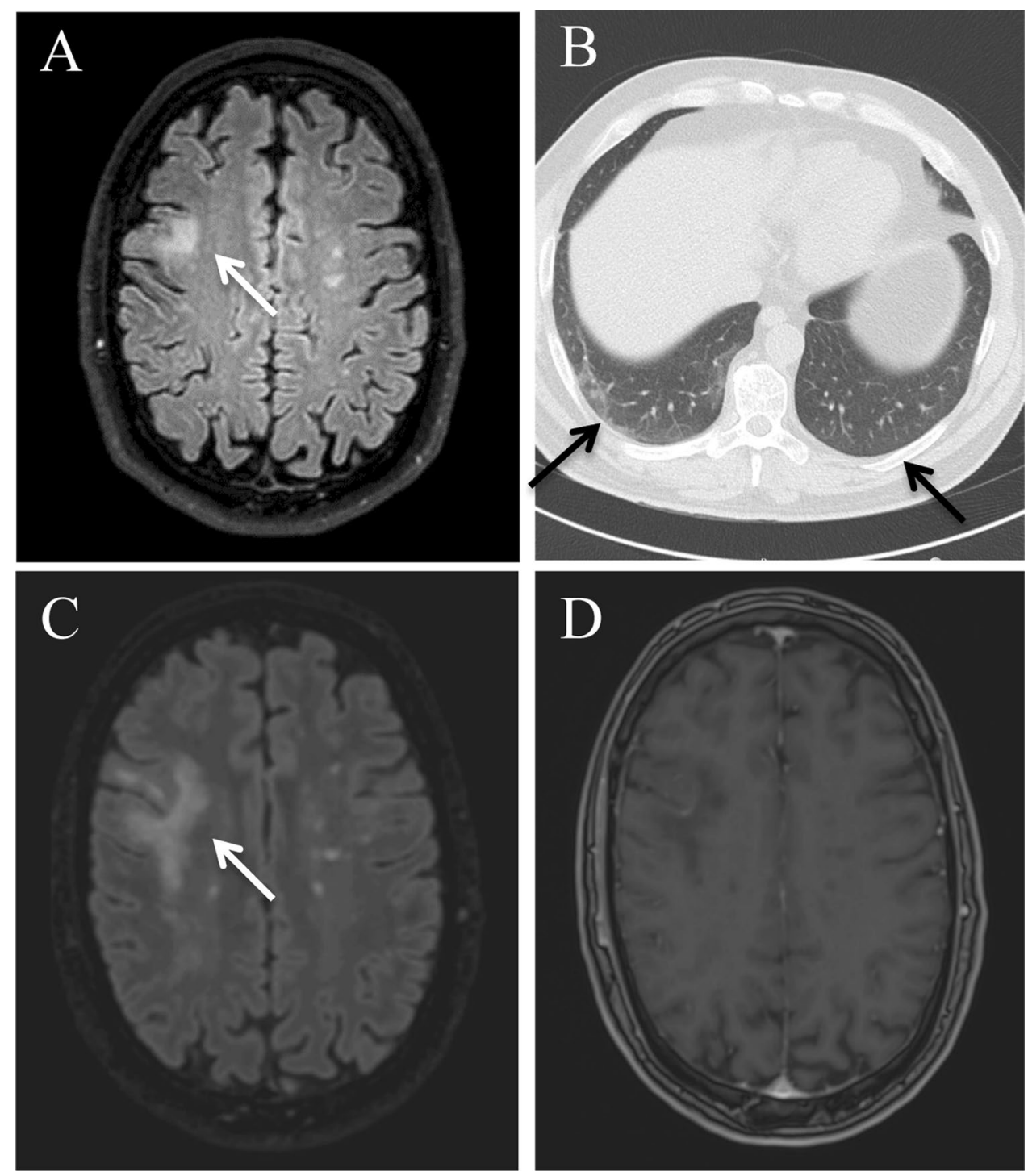

cortical typical MS lesion. Thus, interferon beta-1-a was introduced to treat MS.

The diagnosis of NTZ-related PML at MRI imaging can be challenging at early stages due to the coexistence of MS lesions (Hodel et al. 2016). In our case, the subcortical frontal location, involving the $U$ fibers with sparing of the cortex and ill-defined borders toward the white matter, without gadolinium enhancement, were highly suggestive of PML. After NTZ interruption, the re-establishment of immunocompetence may lead to an excessively overreactive immune response referred to as immune reconstitution inflammatory syndrome (IRIS) (Tan et al. 2011). IRIS can be life-threatening and, in NTZ-treated patients who developed PML, it should be suspected when clinical and radiological (contrast-enhancing lesions with mass effect) worsening occurs 2-5 weeks after NTZ interruption (Tan et al. 2011). In our case, the neurological deterioration occurring more than 2 months after NTZ discontinuation, without imaging inflammatory signs, were thus not suggestive of IRIS (Tan et al. 2011). Even if some imaging inflammatory signs were observed 3 months after NTZ discontinuation, these were more limited than those generally observed during IRIS (Tan et al. 2011). Therefore, we considered, at this later stage, a diagnosis of mild "inflammatory" PML, which has in general a good prognosis (Pasquier and Koralnik 2003), as observed in the patient.

Except for SARS-CoV-2 infection, no other potential factors of PML worsening were identified. Thus, we pose the hypothesis that SARS-CoV-2 was instrumental in the PML deterioration. A first explanation to this deterioration could be the alleged SARS-CoV-2 neurotropism (Zhou et al. 2020). However, we did not demonstrate SARS$\mathrm{CoV}-2$ replication in the CSF, although it must be noted that this analysis was performed only in the second CSF sample, after the resolution of COVID-19. Another mechanism could be the lymphopenia that occurs sometimes 
Fig. 2 Axial brain-MRI demonstrates progressive enlargement of the right frontal lesion on T2/Flair-weighted sequence (a) and appearance of peripheral punctuate T1-weighted postcontrast enhancement (b), from May to June 2020. Axial brainMRI shows reduction in size of the right frontal lesion on $\mathrm{T} 2 /$ Flair-weighted sequence (c) and disappearance of T1-weighthed post-contrast enhancement (d), from July to September 2020
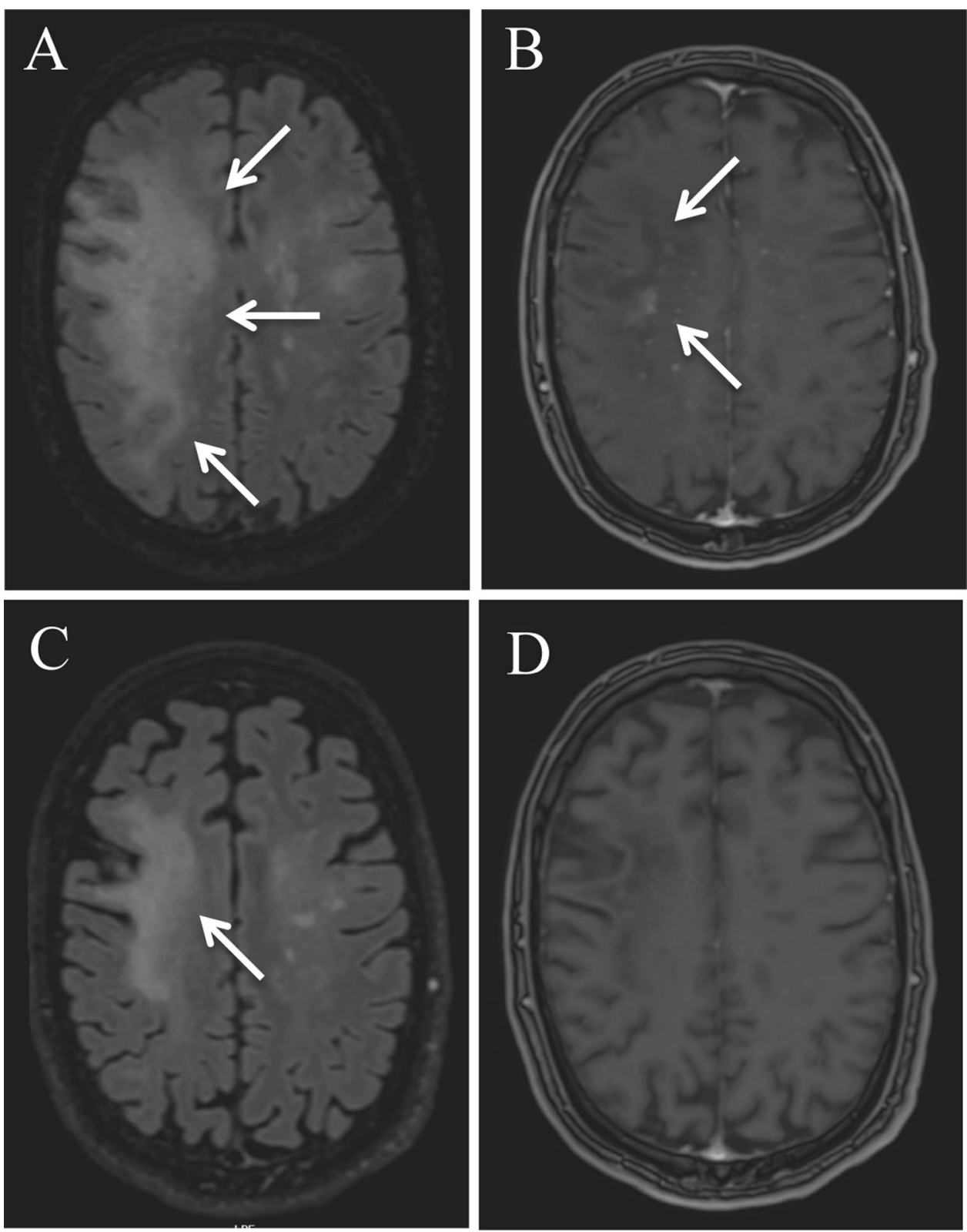

during COVID-19 (Song et al. 2020). Such lymphopenia may have suppressed JCV T-cell response and thus give account of PML progression. Speaking relatively against this hypothesis, we did not find any decrease of lymphocyte count. Nevertheless, even without overt lymphopenia, it is likely that COVID-19 induced some immune dysregulation (Song et al. 2020). This may have been sufficient to affect the cellular immune response, which is crucial for JCV containment (Pasquier et al. 2004). The radiological inflammatory signs seen later on point to a recovered JCV immune response, made possible by the disappearance of the immune disturbances caused by COVID-19. Finally, we can suppose that the neurological deterioration was due to a parainfectious mechanism, as already hypothesized in other CNS manifestations observed during COVID19 (Khateb et al. 2020; Correia et al. 2020; Scoppettuolo et al. 2020). As previously reported in the CSF analysis of COVID-19 patients with neurological symptoms (Tandon et al. 2021), the slight inflammatory alterations (elevated proteins and mild lymphocyte predominant pleocytosis) found in the second CSF analysis of the patient can account for a direct immune-mediated response against the CNS triggered by SARS-CoV-2.

Summarizing, this case raises awareness about the potential role of SARS-CoV-2 on PML evolution. Though its cerebral replication could not be proven, the immune response to SARS-CoV-2 may have contributed to PML progression. 


\section{Declarations}

Conflict of interest The authors declare no competing interests.

\section{References}

Bloomgren G, Richman S, Hotermans C, Subramanyam M, Goelz S, Natarajan A et al (2012) Risk of natalizumab-associated progressive multifocal leukoencephalopathy. N Engl J Med 366(20):1870-1880

Correia AO, Feitosa PWG, Moreira JLS, Nogueira SAR, Fonseca RB, Nobre MEP (2020) Neurological manifestations of COVID-19 and other coronaviruses: as systematic review. Neurol Psychiatry Brain Res 37:27-32

Du Pasquier RA, Koralnik IJ (2003) Inflammatory reaction in progressive multifocal leukoencephalopathy: harmful or beneficial? J Neurovirol 9(Suppl 1):25-31

Du Pasquier RA, Kuroda MJ, Zheng Y, Jean-Jacques J, Letvin NL, Koralnik IJ (2004) A prospective study demonstrates an association between JC virus-specific cytotoxic T lymphocytes and the early control of progressive multifocal leukoencephalopathy. Brain 127(Pt 9):1970-1978

Hodel J, Outteryck O, Dubron C, Dutouquet B, Benadjaoud MA, Duhin E et al (2016) Asymptomatic progressive multifocal leukoencephalopathy associated with natalizumab: diagnostic precision with MR imaging. Radiology 278(3):863-872

Khateb M, Bosak N, Muqary M (2020) Coronaviruses and central nervous system manifestations. Front Neurol 11:715
Polman CH, O’Connor PW, Havrdova E, Hutchinson M, Kappos L, Miller DH et al (2006) A randomized, placebo-controlled trial of natalizumab for relapsing multiple sclerosis. N Engl J Med 354(9):899-910

Rudick RA, Stuart WH, Calabresi PA, Confavreux C, Galetta SL, Radue EW et al (2006) Natalizumab plus interferon beta-1a for relapsing multiple sclerosis. N Engl J Med 354(9):911-923

Scoppettuolo P, Borrelli S, Naeije G (2020) Neurological involvement in SARS-CoV-2 infection: a clinical systematic review. Brain BehavImmun Health 5:100094

Song JW, Zhang C, Fan X, Meng FP, Xu Z, Xia P et al (2020) Immunological and inflammatory profiles in mild and severe cases of COVID-19. Nat Commun 11(1):3410

Tan IL, McArthur JC, Clifford DB, Major EO, Nath A (2011) Immune reconstitution inflammatory syndrome in natalizumab-associated PML. Neurology 77(11):1061-1067

Tandon M, Kataria S, Patel J, Mehta TR, Daimee M, Patel V et al (2021) A comprehensive systematic review of CSF analysis that defines neurological manifestations of COVID-19. Int J Infect Dis 104:390-397

Zhou Z, Kang H, Li S, Zhao X (2020) Understanding the neurotropic characteristics of SARS-CoV-2: from neurological manifestations of COVID-19 to potential neurotropic mechanisms. J Neurol 267(8):2179-2184

Publisher's Note Springer Nature remains neutral with regard to jurisdictional claims in published maps and institutional affiliations. 